\title{
Factors Associated with Survival of Out-of-Hospital Cardiac Arrest by Cardiopulmonary Resuscitation of Bystander in Korea
}

\author{
Hyun-Soo Kim ${ }^{1}$, Jung-Hee Park ${ }^{2}$, Kwang-Hwan Kim ${ }^{3}$, Seok-Hwan Bae ${ }^{4}$, Ju-Dong Jang ${ }^{1}$, Moo-Sik Lee ${ }^{1}$ \\ ${ }^{1}$ Professor, Department of Preventive Medicine, ${ }^{2}$ Assistant Professor, Department of Emergency Medical Service, \\ ${ }^{3}$ Professor, Department of Hospital Management, ${ }^{4}$ Associate Professor, Department of Radiological Science, \\ Konyang University, Daejeon, Korea
}

\begin{abstract}
Background: Out-of-hospital cardiac arrest (OHCA) affects more than 25,000 cases each year in Korea. The rate of survival and neurological recovery tend to increase in Korea, but less than other developed countries. Despite the increase in bystander cardiopulmonary resuscitation (CPR), the number of patients who have an automated external defibrillator (AED) applied by a bystander remains low. We studied the effect of bystander CPR on survival of OHCA in Korea. Methods: This study was conducted using the 'Sudden Cardiac Arrest (SCA) Survey' of Korea Centers for Disease Control \& Prevention (KCDC) in 2009 to 2018. A total of 45,719 cases of OHCA patients were analyzed. We used a chi-square test, log-rank test, multivariable logistic regression and cox-regression analysis to determine the effect of bystander CPR on survival of OHCA, depending on various factors. Results: The rate of survival of OHCA was significantly affected by age, past medical history, initial cardiac rhythm, pre-hospital CPR and defibrillation, transport time and a type of bystander CPR. The survival rate of patients who received bystander CPR was $44.3 \%$, compare with those who did not (43.4\%). In cases where bystander CPR was received, the survival rate of patients with AED (63.1\%) was higher than patients with only chest compression (44.2\%). Conclusions: With the increase in bystander CPR rates, it is difficult to expect the effect of intervention of bystanders in Korea. Even though the education on AED and the devices itself has been spread throughout the country since 2008, the use of AED remains low. Nevertheless, the use of AED has a positive effect on the survival of OHCA. Improvements on the rate of AED use by bystander in Korea needs to be further elaborated on.
\end{abstract}

Keywords: Out-of-hospital cardiac arrest, Cardiopulmonary resuscitation, Survival, Korea

\section{Introduction}

The Korean national death toll rose consecutively each year from 2014 to 2018 then dropped slightly in 2019. The overall causes of death were malignant neoplasm, heart disease, and pneumonia respectively. In particular, heart disease is ranked second in people in

\section{Correspondence:}

\section{Moo Sik Lee}

Department of Preventive Medicine, College of Medicine Konyang University

158, Gwanjeodong-ro, Seo-gu, Daejeon, South Korea their 60's and older, and is included in the top five in all age groups, and is cited as the next most important cause of death after malignant neoplasm. According to the National Statistical Office, the mortality rate of ischemic heart disease, including myocardial infarction and angina is 26.7 per 100,000 people, higher than that of other circulatory diseases in their 40's and 60's, therefore the need for national management is high.

Heart disease is an acute condition that can cause death, and according to the Korea Center for Disease control and Prevention's announcement in 2019, cardiac arrest occurred more than 25,000 times every year since 
2010. The overall standardization rate has been on the rise from 39.3 per 100,000 people in 2006 to 45.4 per 100,000 in 2013 , but has since declined steadily to 39.7 per 100,000 people in 2018, and the survival rate of acute cardiac arrest has steadily increased from $2.3 \%$ in 2006 to $10.0 \%$ in 2018 . The rate of spontaneous circulation recovery before hospital arrival increased from $0.9 \%$ in 2006 to $7.8 \%$ in 2018 , while the rate of the brain function recovery increased from $0.6 \%$ in 2006 to $5.1 \%$ in 2018 . Overall, improvements in cardiopulmonary resuscitation in the general population and spontaneous circulation recovery before arrival at the hospital contributed to improving the survival rate of patients with acute cardiac $\operatorname{arrest}^{[1]}$. Nevertheless, compared to Singapore, which has $17.9 \%$ spontaneous circulation recovery rate, and the United States which has $30.7 \%$, cardiac arrest rates are low, but the final survival results of cardiac arrest patients such as spontaneous circulation recovery rate and brain function recovery are still insufficient ${ }^{[2]}$. The CARES (Cardiac Arrest Registry to Enhance Survival), a cardiac arrest program by the US Center for Disease Control and Prevention, reported a slight increase in cardiac arrest cases with 76.5 per 100,000 people in 2019 compared to the previous year and the circulation recovery rate before arrival at the hospital is $30.7 \%$ while the rate of brain function recovery is $10.5 \%{ }^{[3]}$. Cardiac arrest is caused by a series of conditions, in which blood flow to the tissue is stopped, oxygen, nutrients are cut off, cells are necrotic, and eventually blood is destroyed. Causes of cardiac arrest can be divided into cardiac arrest caused by heart failure and conditions other than heart disease. Defibrillation for ventricular fibrillation is more important than cardiopulmonary resuscitation because heart rate can recover without damage to the body's tissues by 4 to 5 minutes from the time of electrical arrest. It is important to maintain oxygen supply by performing cardiopulmonary resuscitation as the circulation period begins from 4 to 5 minutes to 10 minutes before, and then defibrillation and professional resuscitation should be performed to maintain perfusion pressure ${ }^{[4]}$. Therefore, rapid CPR and defibrillation can greatly improve patient survival in the event of cardiac arrest patients, and outof-hospital cardiac arrest patients need a continuous and essential treatment process to the hospital stage. It has been reported that the factors related to the recovery of pre-hospital circulation of patients with out-of-hospital cardiac arrest are highly related to the sighting of cardiac arrest, cardiopulmonary resuscitation by witnesses and paramedics, and the application of automatic cardiac shock $[5,6]$. In Korea, the implementation rate of eyewitness CPR is steadily increasing, but the implementation rate of defibrillation by ordinary people is still low. The public defibrillation program is already implemented in many countries around the world, and has been proven to be effective, and can be implemented with the introduction of automated external defibrillator (AED) and voice guidance systems. In particular, it is ideal to defibrillate as quickly as possible during the electrical period within 5 minutes of cardiac arrest, especially in patients with cardiac arrest, such as ventricular fibrillation and ventricular tachycardia, faster defibrillation than chest pressure ${ }^{[3,4,7]}$. As part of the defibrillation program for the general public, the installation of AEDs was enacted in 2008, and it was expanded in earnest in 2012 to require the installation of AEDs in multi-use facilities and residential facilities over 500 households. The installation area was expanded in 2014 with the revision of the Enforcement Decree of the Emergency Medical Care Act, but the use of AEDs by the public increased from $0.04 \%$ in 2012 to $0.13 \%$ in 2016 , but is still very low ${ }^{[6]}$.

The steps required to increase the survival rate of cardiac arrest patients are called survival chains, and the role of witnesses, especially the public, who first discovered the patient in four rings except the last one, is very important. The public can quickly deliver cardiac arrest patients to primary responders or emergency medical workers after sighting, help circulation of blood flow under chest pressure before delivery, and restore heartbeat by performing defibrillation on patients with ventricular fibrillation or ventricular tachycardia. Considering the large deviation in the capabilities of the general public, unlike primary responders or emergency medical workers who participate in the survival chain, the importance is even greater. As a result, various efforts 
have been made in various fields, including education and promotion of cardiopulmonary resuscitation since 2011, and the distribution of AEDs, and various studies have been carried out. In particular, many studies have been conducted on survival of patients with cardiac arrest outside the hospital, but most exploratory factors have included various factors from sighting to hospital stage. Survival is complicated by many variables and limited data.

In this study, considering that the role of the general public in the survival chain is the timely performance and effect of cardiopulmonary resuscitation and defibrillation before the delivery of cardiac arrest patients to professionals. Specific purposes are as follows: First, the general characteristics of out-of-hospital cardiac arrest patients are identified for their survival. Second, the effect of factors in the hospital transfer process on the survival rate of patients with cardiac arrest outside the hospital is understood. Third, determine whether the general public has knowledge on CPR or not and how CPR methods affect the survival rate of patients with cardiac arrest outside the hospital.

\section{Methodology}

\section{Subjects of the Study}

The study aims to find out the impact of the general public's CPR implementation on the survival of patients with acute cardiac arrest outside the hospital by utilizing the Korea Disease Control and Prevention Agency's "Acute Cardiac Arrest Survey" data. The acute cardiac arrest investigation, which began in 2008, was conducted on all patients with acute cardiac arrest outside the hospital who were transported by 119 paramedics, and the investigation on data from 2006 to 2018 is currently completed. The survey data were added and modified in 2008, 2009, 2011, and 2015, and were subject to data from 2009 to 2018, except for data from 2008 when major variables were omitted. Of the 273,761 patients surveyed from 2009 to 2018, 55,069 people, excluding "Not Applicable" and "Unidentified", were the first to be selected in the category of whether CPR was performed by the general public. In the question of survival status,
54,080 people were selected for the second time, excluding items such as "Unidentified" and "Do Not Resuscitation" (DNR) before death. Finally, the final 45,719 people were selected as subjects of the study, except for cases where the sighting date or report date was later than the arrival date of the emergency room, and cases where the transfer time took more than 12 hours.

\section{Research Method}

"Acute cardiac arrest investigation" is a data consisting of investigation items and results at each stage depending on the survival chain of cardiac arrest patients. The cardiopulmonary resuscitation effects of the general public in Korea, which are intended to be seen in this study, are comprehensively included not only whether to recover from voluntary circulation before arrival at the hospital, but also the results after professional resuscitation. Assuming that the quality of professional resuscitation is the same, the final dependent variable was classified as 'survive' until the spontaneous circulation was restored before the arriving at the hospital, and the voluntary circulation was not restored in the case of death on arrival (DOA, Dead On Arrival), specialized cardiopulmonary surgery was performed in the emergency room, but it was defined as "death" if it was not voluntarily recovered. The independent variables included gender, age, past medical history, and acute cardiac arrest before arrival at the hospital. Variables in the transport phase included whether cardiopulmonary resuscitation was performed, defibrillation was performed before arrival, and transfer time. The age was divided into "under 50 years old", "50-59 years old", "60-69 years old", "7079 years old" and "80 years old or older", and the past medical history was divided into "cardiac disease" in the case of heart disease, "other diseases" in the case of other diseases without heart disease, and "no disease" in the case of no past medical history. Before arriving at the hospital, acute cardiac arrest ECG findings are ventricular fibrillation and ventricular tachycardia, unclear defibrillation rhythm is "shockable", nonshrinkable electric activity, unclear defibrillation rhythm 
210 Medico-legal Update, October-December 2021, Vol.21, No. 4

is "non-shockable", and other rhythms are classified as 'Other'. Implementation of pre-hospital CPR including survival cases during transfer are classified as "transport with CPR", and in case of transfers without CPR, it is classified as "transport without CPR". If defibrillation is clearly recorded in the medical record and no records are known, they are classified as "no" and "yes" if they are clearly recorded in the medical records. The transfer time was defined as the time it took from the time of cardiac arrest to the time of arrival in the emergency room, and calculated by the division. The time of cardiac arrest was converted to "time of cardiac arrest" based on "witness time", "report time", and "estimated time of fire department", and if the time of conversion was later than "time of emergency room arrival", the data was excluded from the study due to poor reliability. The calculated transport time was classified as "within 10 minutes", "within 11-20 minutes", "within 21-30 minutes", "within 31-40 minutes", "within 41-50 minutes", "within 51-60 minutes", and "more than 1 hour". The main independent variables of interest are whether the general public implements CPR or not, except in cases where paramedics and medical personnel who are working with CPR methods are witnesses. CPR of the general public was divided into "yes", "no", and "CPR" only for chest compressions, "AED" for automated external defibrillation, and "CPR+AED" for both chest compressions and automated external defibrillation.

\section{Method of Analysis}

The analysis of the general characteristics of the subjects, variables in the transport phase, and frequency of major independent variables were conducted, and independent variables and chi-square tests were conducted as dependent variables for survival of patients with cardiac arrest outside the hospital. Kaplan-Meier survival analysis (Log-rank test) was performed for each independent variable, and Cox regression was performed with variables that showed significant differences. The statistical significance level was 0.05 , and the statistical program used was SPSS 24.0 (IBM SPSS 24.0 for window).

\section{Results}

Status and type of by-stander CPR implementation by year, survival rate of out-ofhospital acute cardiac arrest patients by year

Of the 45,719 people surveyed, 27,721 (60.6\%) were administered CPR by the public, with $286(25.9 \%)$ in $2009,374(26.8 \%)$ in 2010, 591 (37.1\%) in 2011, 800 (40.6\%) in 2012, 2,323 (48.7\%), 3,424 (53.9\%), 3,821 $(56.2 \%)$ in 2014, 4,363 (73.5\%), 5,382 (74.6\%) in 2016, and 6,357 (73.9\%) in 2018 where the number increased. The CPR method of the general public was more than $99 \%$ on chest compressions only in all years, and the use of AEDs increased every year, with $0.9 \%$ of the population performing chest compressions and AEDs more than three times higher than 2009.

Of the 45,719 people surveyed, 27,721 (60.6\%) were administered CPR by the public, by year with 286 $(25.9 \%)$ in $2009,659(47.1 \%)$ in 2010, 826 (51.9\%), 1,077 (54.6\%) in 2012, 2,189 (45.9\%), 2,809 (44.3\%), $3,060(45.0 \%)$ in $2015,2,410(40.6 \%)$ in $2016,3,025$ $(42.0 \%)$ in $2017,3,528(41.0 \%)$ in 2018 , The trend was increasing until 2012 and then decreasing again by 2018 (Table 1). 
Table 1. Bystander cardiopulmonary resuscitation and survival of out-of-hospital cardiac arrest patients from 2009 to 2018 in Korea

\begin{tabular}{|c|c|c|c|c|c|c|c|c|c|c|c|c|c|c|}
\hline \multirow{3}{*}{$\begin{array}{l}\text { Years } \\
2009\end{array}$} & \multicolumn{4}{|c|}{$\begin{array}{l}\text { Receive bystander CPR } \\
\qquad(\mathrm{n}=\mathbf{4 5 , 7 1 9 )}\end{array}$} & \multicolumn{6}{|c|}{$\begin{array}{c}\text { Type of bystander CPR } \\
\qquad(n=27,728)\end{array}$} & \multicolumn{4}{|c|}{$\begin{array}{c}\text { Survive } \\
(\mathrm{n}=\mathbf{4 5 , 7 1 9 )}\end{array}$} \\
\hline & \multicolumn{2}{|c|}{ Yes } & \multicolumn{2}{|c|}{ No } & \multicolumn{2}{|c|}{ CPR1) } & \multicolumn{2}{|c|}{ AED } & \multicolumn{2}{|c|}{ CPR+AED } & \multicolumn{2}{|c|}{ Survive } & \multicolumn{2}{|c|}{ Death } \\
\hline & 286 & (25.9) & 818 & (74.1) & 285 & (99.7) & 0 & $(0.0)$ & 1 & $(0.3)$ & 527 & $(47.7)$ & 577 & (52.3) \\
\hline 2010 & 374 & (26.8) & 1,024 & (73.2) & 373 & (99.7) & 0 & $(0.0)$ & 1 & $(0.3)$ & 659 & (47.1) & 739 & $(52.9)$ \\
\hline 2011 & 591 & (37.1) & 1,002 & (62.9) & 588 & (99.5) & 0 & $(0.0)$ & 3 & $(0.5)$ & 826 & (51.9) & 767 & $(48.1)$ \\
\hline 2012 & 800 & (40.6) & 1,171 & (59.4) & 794 & (99.3) & 1 & $(0.1)$ & 5 & $(0.6)$ & 1,077 & (54.6) & 894 & (45.4) \\
\hline 2013 & 2,323 & (48.7) & 2,444 & (51.3) & 2,304 & (99.2) & 6 & $(0.3)$ & 13 & $(0.6)$ & 2,189 & (45.9) & 2,578 & (54.1) \\
\hline 2014 & 3,424 & (53.9) & 2,923 & (46.1) & 3,394 & (99.1) & 7 & $(0.2)$ & 23 & $(0.7)$ & 2,809 & (44.3) & 3,538 & (55.7) \\
\hline 2015 & 3,821 & $(56.2)$ & 2,973 & (43.8) & 3,795 & (99.3) & 7 & $(0.2)$ & 19 & $(0.5)$ & 3,060 & $(45.0)$ & 3,734 & $(55.0)$ \\
\hline 2016 & 4,363 & (73.5) & 1,573 & (26.5) & 4,325 & (99.1) & 2 & $(0.0)$ & 36 & $(0.8)$ & 2,410 & (40.6) & 3,526 & (59.4) \\
\hline 2017 & 5,382 & (74.6) & 1,828 & (25.4) & 5,337 & $(99.2)$ & 5 & $(0.1)$ & 40 & $(0.7)$ & 3,025 & $(42.0)$ & 4,185 & (58.0) \\
\hline 2018 & 6,357 & (73.9) & 2,242 & (26.1) & 6,300 & (99.1) & 0 & $(0.0)$ & 57 & $(0.9)$ & 3,528 & (41.0) & 5,071 & $(59.0)$ \\
\hline Total & 27,721 & (60.6) & 17,998 & (39.4) & 27,495 & $(99.2)$ & 28 & $(0.1)$ & 198 & $(0.7)$ & 20,110 & (44.0) & 25,609 & $(56.0)$ \\
\hline
\end{tabular}

General characteristics of the subjects and the major variables in the transport phase in relation to the impact of survival of acute cardiac arrest patients
Of the 45,719 people surveyed, men accounted for $30,119(65.9 \%)$ and women accounted for 15,600 (34.1\%). By age group, 9,672 people $(21.2 \%)$ were under 50, 8,137 people (17.8\%) were between 50 and 
59, 8,083 people (17.7\%) were between 60 and 69, 10,339 people $(22.6 \%)$ were between 70 and 79 , and 9,488 people $(20.8 \%)$ were over 80 . But for the 15,983 people (35.0\%) who had unknown past medical history in medical records, 6,921 patients with heart disease (23.3\%), 16,190 patients (54.4\%) had other diseases other than heart disease and $6.625(22.3 \%)$ had no disease. In the electrocardiogram before arriving at the hospital, non-shock rhythm was the most common with 23,795 (52.0\%), shock rhythm with 7,209 (15.8\%) and other rhythm with 14,715 (32.2\%). In CPR before arriving at the hospital, 42,411 (92.8\%) were transported with continuous CPR, and 3,308 (7.2\%) were transported without CPR. In addition, 9,514 people (20.8\%) conducted defibrillation before arriving at the hospital and 36,207 (79.2\%) did not defibrillate. As for the transfer time, 7,767 people (17.0\%) within 20 minutes, 18,871 people $(41.3 \%)$ within 20 to 30 minutes, and 19,081 people (41.7\%) who took more than 30 minutes. Cases where CPR was performed by a by-stander is $27,721(60.6 \%)$, and cases where CPR wasn't performed was 17,998 (39.4\%), 27,495 people (99.2\%) with the highest prevalence performed chest compressions only, 28 people $(0.1 \%)$ used only AEDs, and $198(0.7 \%)$ used chest compressions and AEDs together. According to the general characteristics of the subjects in this study, $45.3 \%$ of men and $41.5 \%$ of women had the highest survival rate $(p<0.001), 50.7 \%$ of men aged 50 to 59 and the lowest survival rate at $32.4 \%$ over 80 years of age $(p<0.001)$. In the past medical history, $53.3 \%$ of the population with heart disease was the highest prevalence, $52.4 \%$ of the population without diseases and $47.7 \%$ with other diseases $(p<0.001)$. The electrocardiogram before arriving at the hospital showed that $70.9 \%$ of the population showed the highest survival rate, with $42.0 \%$ presenting with other rhythm and 37.0 $\%$ with the non-shock rhythm $(\mathrm{p}<0.001)$. In terms of key parameters during transportation, continuous CPR was performed in $45.2 \%$ of those who were transported before arriving at the hospital, $27.7 \%$ were transported without CPR $(\mathrm{p}<0.001), 64.9 \%$ were defibrillated before arriving at the hospital, and $38.5 \%$ did not do defibrillation $(\mathrm{p}<0.001)$. In relation to the transportation time, $52.8 \%$ of the people were transported within 20 minutes, followed by $45.5 \%$ within 20 to 30 minutes and $38.9 \%$ for those who took more than 30 minutes, indicating that the longer the transportation time, the lower the survival rate was $(\mathrm{p}<0.001)$. In accordance to the survival rate of patients with acute cardiac arrest whether or not CPR was performed, $44.3 \%$ performed CPR and $43.4 \%$ did not perform CPR showed no statistically significant difference $(\mathrm{p}=0.062)$. However, according to the type of CPR of by-stander, $44.2 \%$ used chest compressions only, $71.4 \%$ used AEDs, and $63.1 \%$ used chest compressions and AEDs together, indicating a high survival rate $(\mathrm{p}<0.001)$ (Table 2$)$.

Table 2. Survival of out-of-hospital cardiac arrest by arrest characteristics from 2009 to 2018 in Korea

\begin{tabular}{|c|c|c|c|c|c|c|c|c|}
\hline \multirow{3}{*}{\multicolumn{2}{|c|}{ Variables }} & \multirow{2}{*}{\multicolumn{2}{|c|}{ Total }} & \multicolumn{4}{|c|}{ Survive of out-of-hospital cardiac arrest } & \multirow{3}{*}{ p-value3) } \\
\hline & & & & \multicolumn{2}{|c|}{ Survive } & \multicolumn{2}{|c|}{ Death } & \\
\hline & & 45,719 & (100.0) & 20,110 & (44.0) & 25,609 & $(56.0)$ & \\
\hline \multirow{2}{*}{ Gender } & Male & 30,119 & (65.9) & 13,635 & $(45.3)$ & 16,484 & $(54.7)$ & \multirow{2}{*}{$<0.001$} \\
\hline & Female & 15,600 & $(34.1)$ & 6,475 & $(41.5)$ & 9,125 & $(58.5)$ & \\
\hline \multirow{5}{*}{ Age (years) } & $<50$ & 9,672 & $(21.2)$ & 4,777 & $(49.4)$ & 4,895 & $(50.6)$ & \multirow{5}{*}{$<0.001$} \\
\hline & $50 \sim 59$ & 8,137 & $(17.8)$ & 4,126 & $(50.7)$ & 4,011 & (49.3) & \\
\hline & $60 \sim 69$ & 8,083 & $(17.7)$ & 3,879 & $(48.0)$ & 4,204 & $(52.0)$ & \\
\hline & $70 \sim 79$ & 10,339 & $(22.6)$ & 4,254 & $(41.1)$ & 6,085 & $(58.9)$ & \\
\hline & $\geq 80$ & 9,488 & $(20.8)$ & 3,074 & (32.4) & 6,414 & (67.6) & \\
\hline
\end{tabular}


Cont... Table 2. Survival of out-of-hospital cardiac arrest by arrest characteristics from 2009 to 2018 in Korea

\begin{tabular}{|c|c|c|c|c|c|c|c|c|}
\hline \multirow{3}{*}{$\begin{array}{c}\text { Past medical } \\
\text { history1) } \\
(\mathrm{n}=29,736)\end{array}$} & Cardiac disease & 6,921 & $(23.3)$ & 3,692 & $(53.3)$ & 3,229 & $(46.7)$ & \multirow{3}{*}{$<0.001$} \\
\hline & Other disease & 16,190 & $(54.4)$ & 7,724 & $(47.7)$ & 8,466 & (52.3) & \\
\hline & No disease & 6,625 & $(22.3)$ & 3,471 & $(52.4)$ & 3,154 & $(47.6)$ & \\
\hline \multirow{3}{*}{ Initial rhythm } & Shockable & 7,209 & $(15.8)$ & 5,113 & $(70.9)$ & 2,096 & (29.1) & \multirow{3}{*}{$<0.001$} \\
\hline & Non-shockable & 23,795 & $(52.0)$ & 8,812 & $(37.0)$ & 14,983 & $(63.0)$ & \\
\hline & Other & 14,715 & $(32.2)$ & 6,185 & $(42.0)$ & 8,530 & $(58.0)$ & \\
\hline \multirow{2}{*}{ Pre-hospital CPR } & with CPR & 42,411 & $(92.8)$ & 19,193 & $(45.2)$ & 23,218 & $(54.8)$ & \multirow{2}{*}{$<0.001$} \\
\hline & without $\mathrm{CPR}$ & 3,308 & $(7.2)$ & 917 & $(27.7)$ & 2,391 & (72.3) & \\
\hline \multirow{2}{*}{$\begin{array}{l}\text { Pre-hospital } \\
\text { defibrillation }\end{array}$} & Yes & 9,514 & $(20.8)$ & 19,193 & $(45.2)$ & 3,338 & $(35.1)$ & \multirow{2}{*}{$<0.001$} \\
\hline & No & 36,207 & $(79.2)$ & 917 & $(27.7)$ & 22,271 & (61.5) & \\
\hline \multirow{3}{*}{$\begin{array}{l}\text { Transport time } \\
\text { (minutes) }\end{array}$} & $<20$ & 7,767 & $(17.0)$ & 4,098 & $(52.8)$ & 3,669 & $(47.2)$ & \multirow{3}{*}{$<0.001$} \\
\hline & $20-29$ & 18,871 & $(41.3)$ & 8,584 & $(45.5)$ & 10,287 & $(54.5)$ & \\
\hline & $\geq 30$ & 19,081 & $(41.7)$ & 7,428 & (38.9) & 11,653 & (61.1) & \\
\hline \multirow{2}{*}{ Bystander CPR } & No & 17,998 & $(39.4)$ & 12,290 & $(44.3)$ & 15,431 & (55.7) & \multirow{2}{*}{0.062} \\
\hline & Yes & 27,721 & $(60.6)$ & 7,820 & $(43.4)$ & 10,178 & (56.6) & \\
\hline \multirow{3}{*}{$\begin{array}{c}\text { Type of bystander } \\
\text { CPR }\end{array}$} & CPR2) & 27,495 & $(99.2)$ & 12,145 & $(44.2)$ & 15,350 & $(55.8)$ & \multirow{3}{*}{$<0.001$} \\
\hline & AED & 28 & $(0.1)$ & 20 & (71.4) & 8 & (28.6) & \\
\hline & $\mathrm{CPR}+\mathrm{AED}$ & 198 & $(0.7)$ & 125 & $(63.1)$ & 73 & (36.9) & \\
\hline
\end{tabular}

1) excluding $15,983(35.0 \%)$ missing value

2) only chest compression

3) by chi-square test

CPR; cardiopulmonary resuscitation, AED; automated external defibrillator

\section{Comparison of survival time of acute cardiac arrest patients by factor}

In the survival analysis according to gender, men had 45.50 \pm 0.51 minutes (95\% CI; 44.51-46.49) and women $42.47 \pm 0.92$ minutes (95\% CI; 40.68-44.27) with longer survival times in men $(\mathrm{p}<0.001)$. According to age, $50.81 \pm 1.35$ minutes $(95 \% \mathrm{CI}$; 48.16-53.46) for those under 50 years of age, $50.98 \pm 1.27$ minutes $(95 \% \mathrm{CI}$; 48.49-53.47) for those between 60 and $69,46.22 \pm 0.85$ minutes (95\% CI; 44.55-47.89), $40.97 \pm 0.87$ minutes (95\% CI; 39.27-42.67) for ages 70-79 and 36.64 \pm 0.49 minutes (95\% CI; 35.67-37.61) for ages 80 and older, the lower the age group, the longer the survival time $(\mathrm{p}<0.001)$. In terms of past medical history, $47.54 \pm 1.08$ minutes (95\% CI; 45.43-49.65), 47.23 \pm 1.42 minutes (95\% CI; 44.46-50.01) for heart disease, and $47.23 \pm 1.42$ minutes (95\% CI; 44.46-0.01) with other diseases, 54.21 \pm 2.16 minutes (95\% CI; 49.98-58.43) for absence of a disease, and the survival time was longer in the absence of a disease $(p<0.001)$. Prior to arrival in the hospital, those who spent $64.29 \pm 2.18$ minutes $(95 \%$ CI; 60.01-68.56) presented with shockable rhythm, $39.19 \pm 0.41$ minutes $(95 \% \mathrm{CI}$; 38.38-39.99) with nonshockable rhythm, and $45.12 \pm 0.68$ minutes $(95 \% \mathrm{CI}$; 43.79-46.45) for other rhythms, indicating that patients with cardiac arrest with shockable rhythm had a long survival time $(\mathrm{p}<0.001)$. The group that performed CPR continuously before arriving at the hospital spent 44.48 \pm 0.53 minutes (95\% CI; 43.45-45.52), the group that transported without cardiopulmonary resuscitation spent $41.09 \pm 0.97$ minutes (95\% CI; 39.19-42.99), and the time of survival was longer in the group that performed CPR continuously before transportation $(p<0.001)$. The duration of defibrillation before arrival at the hospital 
was $60.03 \pm 2.09$ minutes (95\% CI; 55.94-64.12) in the group that defibrillated, and $41.15 \pm 0.37$ minutes $(95 \%$ CI; 40.42-41.89 in the group that did not defibrillate. $44.34 \pm 0.76$ minutes ( $95 \% \mathrm{CI}$; $42.85-45.84$ minutes) and $44.06 \pm 0.47$ minutes (95\% CI; 43.14-44.98), depending on whether or not cardiopulmonary resuscitation was performed $(p<0.001)$ and 43.7 minutes $(p<0.001)$ in case of cardiopulmonary resuscitation the survival time in longer $(\mathrm{p}<0.001)$ (Table 3).

Table 3. The Kaplan-Meier estimate for out-of-hospital cardiac arrest from 2009 to 2018 in Korea

\begin{tabular}{|c|c|c|c|c|c|c|c|}
\hline \multicolumn{2}{|c|}{ Variables } & \multicolumn{3}{|c|}{ Mean \pm SD } & \multicolumn{2}{|c|}{$95 \% \mathrm{CI}$} & p-value ${ }^{3)}$ \\
\hline \multirow[b]{2}{*}{ Gender } & Male & 45.50 & \pm & 0.51 & (44.51 & - 46.49$)$ & \multirow{2}{*}{$<0.001$} \\
\hline & Female & 42.47 & \pm & 0.92 & $(40.68$ & - 44.27$)$ & \\
\hline \multirow{5}{*}{ Age (years) } & $<50$ & 50.81 & \pm & 1.35 & $(48.16$ & - 53.46) & \multirow{5}{*}{$<0.001$} \\
\hline & $50 \sim 59$ & 50.98 & \pm & 1.27 & $(48.49$ & $-53.47)$ & \\
\hline & $60 \sim 69$ & 46.22 & \pm & 0.85 & $(44.55$ & - 47.89$)$ & \\
\hline & $70 \sim 79$ & 40.97 & \pm & 0.87 & $(39.27$ & - 42.67$)$ & \\
\hline & $\geq 80$ & 36.64 & \pm & 0.49 & (35.67 & $-37.61)$ & \\
\hline \multirow{3}{*}{$\begin{array}{l}\text { Past medical history }{ }^{1)} \\
(\mathrm{n}=29,736)\end{array}$} & Cardiac disease & 47.54 & \pm & 1.08 & $(45.43$ & - 49.65) & \multirow{3}{*}{$<0.001$} \\
\hline & Other disease & 47.23 & \pm & 1.42 & $(44.46$ & $-50.01)$ & \\
\hline & No disease & 54.21 & \pm & 2.16 & $(49.98$ & $-58.43)$ & \\
\hline \multirow{3}{*}{ Initial rhythm } & Shockable & 64.29 & \pm & 2.18 & $(60.01$ & $-68.56)$ & \multirow{3}{*}{$<0.001$} \\
\hline & Non-shockable & 39.19 & \pm & 0.41 & $(38.38$ & - 39.99) & \\
\hline & Other & 45.12 & \pm & 0.68 & $(43.79$ & - 46.45$)$ & \\
\hline \multirow{2}{*}{ Pre-hospital CPR } & with CPR & 44.48 & \pm & 0.53 & $(43.45$ & - 45.52$)$ & \multirow{2}{*}{$<0.001$} \\
\hline & without CPR & 41.09 & \pm & 0.97 & $(39.19$ & - 42.99) & \\
\hline \multirow{2}{*}{$\begin{array}{l}\text { Pre-hospital } \\
\text { defibrillation }\end{array}$} & Yes & 60.03 & \pm & 2.09 & $(55.94$ & - 64.12) & \multirow{2}{*}{$<0.001$} \\
\hline & No & 41.15 & \pm & 0.37 & $(40.42$ & - 41.89 ) & \\
\hline \multirow{2}{*}{ Bystander CPR } & No & 44.34 & \pm & 0.76 & $(42.85$ & - 45.84) & \multirow{2}{*}{$<0.001$} \\
\hline & Yes & 44.06 & \pm & 0.47 & $(43.14$ & $-44.98)$ & \\
\hline \multirow{3}{*}{$\begin{array}{c}\text { Type of bystander } \\
\text { CPR }\end{array}$} & $\mathrm{CPR}^{2)}$ & 43.77 & \pm & 0.46 & $(42.87$ & - 44.66$)$ & \multirow{3}{*}{$<0.001$} \\
\hline & AED & 33.17 & \pm & 1.63 & $(29.97$ & $-\quad 36.37)$ & \\
\hline & $\mathrm{CPR}+\mathrm{AED}$ & 90.04 & \pm & 11.56 & $(67.38$ & - 112.70) & \\
\hline
\end{tabular}

1) excluding $15,983(35.0 \%)$ missing value

2) only chest compression

3) by chi-square test

CPR; cardiopulmonary resuscitation, AED; automated external defibrillator

\section{Cox Regression Results}

According to the survival analysis of patients with acute cardiac arrest, women had longer survival times $(p<0.001), 1.36$ times for those under 50, 1.32 times for those between 60 and 69, 1.32 times for those between 60 and 69, 1.24 times longer for those between 70 and 79
( $p<0.001)$. Pre-hospital ECG findings showed 1.45 times longer shockable rhythms than non-shockable rhythms, shorter survival times for other rhythms, and statistically significant differences $(p<0.001)$. CPR was continuously performed and transported 1.69 times longer $(p<0.001)$ than without cardiopulmonary resuscitation $(\mathrm{p}=0.003)$ and 1.08 times longer than defibrillation was performed before hospital arrival $(p=0.003)$. According to whether or not CPR was performed by the general public, the survival time was shorter than that without CPR $(\mathrm{OR}=0.84)$ Statistically significant differences $(\mathrm{p}<0.001)$ (Table 4). 
Table 4. Cox proportional hazard regression analysis for survival outcomes of out-of-hospital cardiac arrest in 2009 to 2018 in Korea

\begin{tabular}{|c|c|c|c|c|c|c|}
\hline \multicolumn{2}{|c|}{ Variables } & OR & \multicolumn{3}{|c|}{$95 \% \mathrm{CI}$} & p-value3) \\
\hline \multirow{2}{*}{ Gender } & \multirow{2}{*}{$\begin{array}{c}\text { Female } \\
\text { Male }\end{array}$} & \multirow{2}{*}{$\begin{array}{l}1.00 \\
0.87\end{array}$} & \multirow[b]{2}{*}{$(0.85$} & \multirow[b]{2}{*}{-} & \multirow[b]{2}{*}{$0.90)$} & \\
\hline & & & & & & $<0.001$ \\
\hline \multirow{5}{*}{ Age (years) } & $\geq 80$ & 1.00 & & & & \\
\hline & $<50$ & 1.36 & $(1.30$ & - & 1.43) & $<0.001$ \\
\hline & $50 \sim 59$ & 1.32 & $(1.26$ & - & 1.39) & $<0.001$ \\
\hline & $60 \sim 69$ & 1.32 & $(1.26$ & - & 1.39) & $<0.001$ \\
\hline & 70 79 & 1.24 & (1.18 & - & 1.30) & $<0.001$ \\
\hline \multirow{3}{*}{ Initial rhythm } & Non-shockable & 1.00 & & & & \\
\hline & Shockable & 1.45 & $(1.37$ & - & 1.53) & $<0.001$ \\
\hline & Other & 0.85 & $(0.82$ & - & $0.88)$ & $<0.001$ \\
\hline \multirow{2}{*}{ Pre-hospital CPR } & Without CPR & 1.00 & & & & \\
\hline & With CPR & 1.69 & $(1.58$ & - & 1.81) & $<0.001$ \\
\hline \multirow{2}{*}{$\begin{array}{l}\text { Pre-hospital } \\
\text { defibrillation }\end{array}$} & No & 1.00 & & & & \\
\hline & Yes & 1.08 & (1.03 & - & 1.14) & 0.003 \\
\hline \multirow{2}{*}{ Bystander CPR } & No & 1.00 & & & & \\
\hline & Yes & 0.84 & $(0.81$ & - & $0.86)$ & $<0.001$ \\
\hline
\end{tabular}

\section{Discussion}

This study was conducted from 2009 to 2018 on outof-hospital patients with cardiac arrest to find out how much CPR in the public affects patients' survival. Overall, it is very positive that the number ofCPR implementations by the general public is steadily increasing than before. This seems to be the result of continuous social interest and effort. Since 2011, Korea has gradually expanded the number of compulsory cardiopulmonary resuscitation education subjects. Cardiopulmonary resuscitation education was conducted for various institutions and subjects, and the installations of AEDs were expanded from 2012. In the emergency medical system, the number of ambulances and paramedics has increased significantly, and there is also a system that allows CPR to be ordered through emergency medical phone counselors. Unlike the frequency of CPR by the general public and the mandatory installation of AEDs in public places, the actual use of AEDs by the general public is not increasing significantly. The importance of using AEDs has already been demonstrated ${ }^{[8]}$, and there are reports that community-oriented defibrillator operations improve the survival rate of cardiac arrest patients ${ }^{[9]}$. Existing studies have also reported a high possibility of survival discharge if CPR was performed by the public and defibrillation was performed quickly before hospital arrival ${ }^{[10]}$. Therefore, more research 
is needed in Korea on ways that by-standers can use AEDs. Gender showed no statistically significant difference as a factor in survival rates for out-of-hospital cardiac arrest patients, as in previous studies ${ }^{[11]}$. Other factors showed statistically significant differences, consistent with previous studies, the younger the patient was, the continuous $\mathrm{CPR}$ and defibrillation prior to hospital arrival, and the shorter the transfer time, the higher the survival rate of cardiac arrest patients ${ }^{[12-16]}$. However, cardiopulmonary resuscitation in the general population was found to be unrelated to the survival of patients, unlike previous studies ${ }^{[10]}$. It is already widely known that rapid CPR and rapid defibrillation are very important for patient survival and prognosis in cardiac arrest patients, and medical systems and CPR education are provided for timely response in Korea and many countries. In hospitals with high frequency of cardiac arrest patients, a dedicated medical team exists and is operated. However, unlike cardiac arrest in hospitals, cardiac arrest outside the hospital is unpredictable and there is no infrastructure for blood circulation and heart rate recovery in patients with cardiac arrest. In the United States, the survival rate of cardiac arrest patients in the community is less than $6 \%$, while the survival rate of cardiac arrest patients in hospitals is less than $24 \%{ }^{[17]}$. Since the first witness of cardiac arrest is more likely to be an ordinary person than a medical person, the survival rate of patients with cardiac arrest outside the hospital depends on the ability of members of the community and the emergency medical system. ${ }^{[9,18]}$. Existing studies have also reported an increase in the rate of survival as the educational experience of CPR increases ${ }^{[19]}$. Nevertheless, the results of the study suggest that the low effect of CPR on the general public is due to the transfer time. If the heart rate recovers 4 to 5 minutes after cardiac arrest, it can be recovered without damage to the body's tissues, and it is important to maintain oxygen supply by CPR before 10 minutes, and after 10 minutes, defibrillation and professional resuscitation should be performed to maintain perfusion pressure. In line with this, cardiopulmonary resuscitation in the general public is limited to within 10 minutes, and more than that, the influence will be minimal. In this study, the transfer time was calculated in minutes, and $17 \%$ of the cases where the transfer time is within 20 minutes, and $1.9 \%$, if reduced to less than 10 minutes, the impact of cardiopulmonary resuscitation is expected to be less. The arrhythmia of impact rhythm, such as ventricular fibrillation and ventricular fibrillation, is found in 60$85 \%$ of cardiac arrest patients ${ }^{[4]}$, and is likely to recover only defibrillation. Conventional cohort studies have also shown that automatic cardiac arresters are limited in survival in patients with early cardiac rhythms ${ }^{[20]}$, and that CPR by the public and the use of automatic cardiac arresters are independently related to survival. There are also reports of high survival rates in patients with the same shock rhythm when defibrillation is performed early ${ }^{[3]}$. In line with these existing studies, this study also showed significant differences in survival rates depending on how the general population performs CPR. Patients with defibrillation were 1.7 times more likely to survive than those with only chest pressure. This is believed to be because if the heartbeat recovers within five minutes of cardiac arrest, there is a chance to recover without damage. Nevertheless, Korea's use of automatic cardiac arrestors is less than 1 percent. Therefore, it is necessary to educate and promote automatic cardiac arrestors so that they can be easily used by the general public, along with the mandatory supply and installation of automatic cardiac arrestors. The limitations of this study were, first, limited in the composition and selection of variables by utilizing secondary data, and there were many manipulative definitions and assumptions by reprocessing and utilizing the data. In particular, there were many cases where the arrival date in the emergency room was excluded from the subjects before the witness date or report date, and there was no information from the general public who performed early CPR. Second, it was not possible to directly compare and analyze the characteristics of first aid workers and hospitals visiting. Existing studies have shown that factors at the hospital level have a positive effect on the survival of out-ofhospital cardiac arrest patients ${ }^{[21]}$. In this study, it is assumed that the factors at the hospital stage will be the same, and if it is voluntarily recovered after CPR after arrival at the hospital, it is classified as survival in the 
resulting variable, so attention is needed in the analysis of the results. However, if the utilization of AEDs increases in the future and the number of research cases increases, it is believed that the factors at the hospital stage need to be examined in detail. Third, during survival analysis, the transfer time is assumed to be survival time, which may differ from the actual survival and death time. The definition of transfer time in this study was replaced by the time from the initial sighting to the arrival time of the emergency room, and the report time was based on the time of reporting if the sighting was unclear or missing. Therefore, the time between survival and death after cardiac arrest may vary. However, there is no significant difference between sighting and reporting time within the research data of this study, and the actual transfer time reflects the survival time of patients with cardiac arrest outside the hospital. Korea's emergency medical system has been developed and improved, and awareness and implementation of CPR has steadily increased due to the efforts and interest of the local community and securing emergency medical resources, we now need to pay attention to practical use. As the results of this study show, CPR in the general population is increasing, but its effectiveness is difficult to expect, and unlike the dissemination and training of AEDs, it is not well utilized. In the future, research on the use of AEDs is expected to be needed in CPR.

\section{Conclusion}

Since 2010, more than 25,000 cardiac arrest cases have occurred every year, and the survival rate and recovery rate of acute cardiac arrest have continued to improve. However, compared to major developed countries, the rate of self-circulating recovery or brain function recovery is still insufficient. The rate of cardiopulmonary resuscitation in witnesses has increased steadily, but the rate of defibrillation is low. As cardiopulmonary resuscitation of witnesses, especially primary responders, is important in the survival chain, it is necessary to determine the impact of cardiopulmonary resuscitation on patients with acute cardiac arrest.
Men had a lower survival rate than women, and if they showed an electrocardiogram of shock rhythm, continued CPR before arrival, and defibrillation, the earlier the transfer time, the higher the survival rate of patients with acute cardiac arrest. The actual CPR of the general public is increasing, but the effect is not visible, and unlike the distribution and education of automatic cardiac shock devices, it is not being used well. Nevertheless, since the use of automatic cardiac arrest devices has a positive effect on the survival of patients with acute cardiac arrest, it is deemed necessary to study the use of automatic cardiac arrest devices in future CPR.

\section{Source Funding: None.}

\section{Conflict of Interest : None.}

Ethical Clearance : This study was prepared using secondary data on acute cardiac arrest by the Korea Disease Control and Prevention Agency, and was subject to IRB exemption.

\section{References}

1. Yoon HJ, Park SJ, Ahn JY, Lee YK. Key findings of the sudden cardiac arrest (SCA) survey from 2006 to 2018. Public Health Weekly Report 2020;13(1):77-90. KMID:1143420200130010077

2. Eng Hock Ong M, Chan YH, Anantharaman V, Lau ST, Lim SH, Seldrup J. Cardiac arrest and resuscitation epidemiology in Singapore (CARE I study). Prehosp Emerg Care 2003;7(4):427-33. doi: 10.1080/312703002120. PMID: 14582091.

3. CARES. Cardiac Arrest Registry to Enhance Survival. CARES Annual Report 2019.

4. Korea Association ofCardiopulmonary Resustation. Basic Cardiopulmonary Resuscitation, Koonja Publisher, 2018

5. Jung Sk, Kang HD, O MS, Song JS, Oh SH. Resuscitation: Comprehensive review of prehospital factors associated with field return of spontaneous circulation after out-of hospital cardiac arrest in one province, J Korean Soc Emerg Med 2016;27(1):98-106.

6. Lee YA. Influential factors on phased survival after sut-of hospital cardiac arrests; based on the 'Acute Cardiac Arrest Survey of Korea Centers for Disease 
Control \& Prevention, Dissertation of Graduate School, Jeju National University, 2019.

7. Cummins RO. From concept to standard-ofcare? Review of the clinical experience with automated external defibrillators. Ann Emerg Med 1989 ;18(12):1269-75. doi: 10.1016/s01960644(89)80257-4. PMID: 2686497.

8. Atwood C, Eisenberg MS, Herlitz J, Rea TD. Incidence of EMS-treated out-of-hospital cardiac arrest in Europe. Resuscitation 2005;67(1):75-80. doi: 10.1016/j.resuscitation.2005.03.021. PMID: 16199289.

9. Stiell IG, Wells GA, Field BJ, Spaite DW, De Maio VJ, Ward R, Munkley DP, Lyver MB, Luinstra LG, Campeau T, Maloney J, Dagnone E. Improved out-of-hospital cardiac arrest survival through the inexpensive optimization of an existing defibrillation program: OPALS study phase II. Ontario Prehospital Advanced Life Support. JAMA 1999:281(13):1175-81. doi: 10.1001/ jama.281.13.1175. PMID: 10199426.

10. Jung SY, Kim CW, Hong SO. The factors influencing survival of Out-of-hospital Cardiac Arrest with cardiac etiology, JKAIS 2016;17(2):560-69.

11. DickerB, ConaglenK, Howie G. Genderand survival from out-of-hospital cardiac arrest: a New Zealand registry study. Emerg Med J 2018 ;35(6):367-71. doi: 10.1136/emermed-2017-207176. Epub 2018 Apr 16. PMID: 29661780.

12. Kim SH. Factors affecting recovery of spontaneous circulation in patients before cardiac arrest in emergency department : 2012 2016 focused on medical records data, Korean J Med Law 2018;19(2):209-33.

13. Park IS, Kim EJ, Sohn HS, Kang SH. Factors influencing the return of spontaneous circulation of patients with out-of-hospital cardiac arrest. Journal of Digital Policy \& Management 2013;11(9):22938.

14. Lopez-Messa, J.B., J.I. Alonso-Fernandez, J.M. Andres-de Llano, J.R. Garmendia-Leiza, J. Ardura-Fernandez, F. de Castro-Rodriguez and J.M. Gil-Gonzalez. General characteristics out of hospital cardiac arrest of cardiac origin recorded by an emergency medical service. Emergencias 2012;24(1):28-34.
15. May S, Zhang L, Foley D, Brennan E, O’Neil B, Bork E, Levy P, Dunne R. Improvement in NonTraumatic, Out-Of-Hospital Cardiac Arrest Survival in Detroit From 2014 to 2016. J Am Heart Assoc 2018;21;7(16):e009831. doi: 10.1161/ JAHA.118.009831. PMID: 30369308; PMCID: PMC6201400.

16. Bürger A, Wnent J, Bohn A, Jantzen T, Brenner S, Lefering R, Seewald S, Gräsner JT, Fischer M. The Effect of Ambulance Response Time on Survival Following Out-of-Hospital Cardiac Arrest. Dtsch Arztebl Int 2018;20;115(33-34):541-48. doi: 10.3238/arztebl.2018.0541. PMID: 30189973; PMCID: PMC6156551.

17. Becker LB, Aufderheide TP, Graham R. Strategies to Improve Survival From Cardiac Arrest: A Report From the Institute of Medicine. JAMA 2015;21;314(3):223-4. doi: 10.1001/ jama.2015.8454. PMID: 26132709.

18. Hollenberg J, Herlitz J, Lindqvist J, Riva G, Bohm $\mathrm{K}$, Rosenqvist M, Svensson L. Improved survival after out-of-hospital cardiac arrest is associated with an increase in proportion of emergency crewwitnessed cases and bystander cardiopulmonary resuscitation. Circulation 2008;22;118(4):389-96. doi: $10.1161 /$ CIRCULATIONAHA.107.734137. Epub 2008 Jul 7. PMID: 18606920.

19. Ro YS, Shin SD, Song KJ, Hong SO, Kim YT, Lee DW, Cho SI. Public awareness and self-efficacy of cardiopulmonary resuscitation in communities and outcomes of out-of-hospital cardiac arrest: A multilevel analysis. Resuscitation 2016;102:17-24. doi: 10.1016/j.resuscitation. 2016.02.004. Epub 2016 Feb 17. PMID: 26898411.

20. Nas J, Thannhauser J, Herrmann JJ, van der Wulp $\mathrm{K}$, van Grunsven PM, van Royen N, de Boer MJ, Bonnes JL, Brouwer MA. Changes in automated external defibrillator use and survival after outof-hospital cardiac arrest in the Nijmegen area. Neth Heart J 2018;26(12):600-5. doi: 10.1007/ s12471-018-1162-9. PMID: 30280320; PMCID: PMC6288040.

21. Shin WM, Kim JM, Park CH, Shin E, Tchoe B. Analysis of factors influencing the survival of patients with Out-of-Hospital of Cardiac Arrest. Korean Public Health Research 2020;46(2):93-105. 